\title{
Tejido y educación ambiental en comunidades kichwas de Tena, Ecuador
}

Recibido: 5 de julio 2018

Revisado: 12 de julio 2018

Aprobado: 1 de agosto 2018

Caroline Nicole Oriana Bacquet Pérez

Chilena. Doctora en

Bioquímica. Docente e investigadora titular y coordinadora de Investigación de la Universidad Regional

Amazónica Ikiam, Tena,

Ecuador. Especialista en epigénita, interesada en usar el tejido como herramienta de difusión y de transferencia científica.

Correo electrónico: linbacquet@gmail.com

\section{Jorge Alejandro Batres Quevedo}

Guatemalteco. Doctor en Ciencias Sociales. Miembro de

la Red de Investigadores Adjuntos del Departamento Ecuménico de Investigación, DEI, Costa Rica. Especialista en Psicología social, interesado en temas de educación ambiental e identidad sociocultural.

Correo electrónico: batresquevedo@gmail.com
Resumen: Este artículo se originó en una experiencia con esas características: tejer animales amazónicos con mujeres kichwas, como una estrategia de educación y de conservación ambiental, sin encuadre educativo formal, objetivos predefinidos o metas preestablecidas. A medida que el proceso avanzó, fue mostrando otras utilidades: posibilidades de emprendimiento productivo, generación de un ambiente propicio para múltiples aprendizajes, desarrollo de vínculos entre mujeres e interrelación entre la conservación de la diversidad biológica y la cultural. Por ser un proceso abierto, no pueden compartirse conclusiones de conocimiento, pero sí experiencias y aprendizajes que pueden servir de referencia para proyectos similares.

Palabras clave: Educación ambiental; tejido; biocentrismo; cultura kichwa

\section{Knitting and Environmental Education in Kichwa Communities of Tena, Ecuador}

Abstract: This article describes such an experience: knitting Amazonian animals with Kichwa women, as a strategy for education and environmental conservation; without any formal framework, predefined objectives or preestablished goals. As the process developed, it also showed a potential for productive entrepreneurship, acquiring of soft skills, development of bonds between women, and interrelations between conservation of biodiversity and culture. Because it is an open process, theoretical conclusions have not been produced; however, the experiences and lessons learned can serve as a reference for similar initiatives.

Key words: Environmental education; knitting; biocentrism; kichwa culture 


\section{Introducción}

“Caminábamos (...) con el alma abierta al mundo, curiosos, receptivos, por las sendas (...) donde temprano se aprende que solo a costa de mucha testarudez se consigue tejer la vida" (Freire, Pedagogía de la autonomía 2004, 34).

La educación ambiental, generalmente, se asocia a la protección de la naturaleza, lo cual supone el aprendizaje de conceptos especializados, así como valores y prácticas de conservación. En las definiciones tradicionales de educación ambiental, se observa esta idea; por ejemplo, en el principio 19 de la Declaración de Estocolmo:

Es indispensable una educación en valores ambientales (...) para ensanchar las bases de una opinión pública bien informada y de una conducta de los individuos, de las empresas y de las colectividades, inspirada en el sentido de su responsabilidad en cuanto a la protección y mejoramiento del medio en toda su dimensión humana (ONU 1972, 5).

Lo mismo se aprecia cuando se define la educación ambiental en términos más precisos, es decir, prevalece la misma concepción. En el Congreso Internacional de Educación y Formación sobre el Medio Ambiente, se entiende la educación ambiental como:

un proceso permanente en el cual los individuos y las comunidades adquieren conciencia de su medio y aprenden los conocimientos, los valores, las destrezas, la experiencia y también la determinación que les capacite para actuar individual y colectivamente en la resolución de los problemas ambientales presentes y futuros (UNESCO 1987, 11).

A partir de los años 1990, se ponen de moda los conceptos de sostenibilidad y sustentabilidad, lo cual se refleja, de inmediato, en la definición de educación ambiental; sin embargo, el concepto tradicional no cambia fundamental- 
mente. Por ejemplo, la llamada educación para el desarrollo sostenible integra los principios y las prácticas de desarrollo sostenible en la educación y aprendizaje. Otra vez, se trata de cambios de conocimiento, valores y actitudes, ahora destinados a construir una sociedad más sostenible (UNESCO 2017).

Entre las múltiples críticas que se han hecho a la noción tradicional de la educación ambiental, para efectos de este artículo, se resaltarán tres: primero, reproduce una visión antropocéntrica; segundo, corresponde a una educación ambiental institucionalizada; y tercero, está escolarizada. En seguida, se discutirá, de forma breve, cada uno de estos aspectos.

En primer lugar, en la concepción tradicional de educación ambiental se reproduce la dicotomía ser humano-naturaleza, en la cual el ser humano se coloca por fuera de la naturaleza, como sujeto observador, con capacidad de abstraerla como objeto de conocimiento (Fogar 2013, García 2002). Desde este lugar, que a veces es el centro, el ser humano concibe a la naturaleza como objeto, como recurso disponible para producir su bienestar.

Eduardo García (2002) advierte que esta concepción es antropocéntrica y paternalista. Según él, produce una educación sobre el ambiente y para el ambiente, en la medida en que lo concibe como un recurso y su finalidad es conservarlo y protegerlo; pero no por el ambiente mismo, sino porque es un bien necesario para la productividad humana.

La idea tradicional de la educación ambiental se reproduce, sobre todo, en la academia. Es probable que esta sea una de las razones por las cuales la educación ambiental se ha convertido en una especialidad de académicos; de ecólogos, más específicamente. Ahora bien, esta concepción también es asumida por otros actores institucionales, UNESCO y ONU, por ejemplo, además de las ONG y de los movimientos ambientalistas alrededor del mundo.

El activismo de la educación ambiental abarca seminarios, congresos, campañas, encuentros, firma de acuerdos, talleres y más recientemente cursos especializados en instituciones educativas. Los objetivos que se proponen en estas actividades son diversos, van desde la formación conceptual, pasan por el desarrollo de capacidades, hasta la generación de actitudes. Pero todas tienen en común una preocupación creciente por el deterioro de las condiciones del medio ambiente y sus recursos: calentamiento global, deforestación, contaminación ambiental, etc.

El conjunto de actores mencionados y sus acciones características han producido una institucionalización de la educación ambiental. Esta se basa en un optimismo excesivo en la escolarización de la educación ambiental y las posibilidades de la ciencia y de la tecnología en la solución de la problemática ambiental. De acuerdo con Edgar González y Miguel Ortega (2009), tal escolarización es un acto fallido, pues ocurre en el marco de un agotamiento de los sistemas escolares y produce un enfoque ambientalista y focalizado 
en valores y actitudes, sin tener claros aspectos económicos, políticos e ideológicos que determinan hoy en día el estado del medio ambiente.

En el presente artículo, se discute una experiencia de educación ambiental orientada hacia una práctica concreta: tejer animales amazónicos como estrategia de educación y conservación ambiental. La experiencia que se relata coincide con las motivaciones de Eduardo García y otros autores (García 2002, Caride y Meira 2001, Breiting 1997, Gutiérrez 1995), en cuanto a generar una educación ambiental que capacite para la acción y la resolución de conflictos ambientales, desde la acción misma.

Todo proceso que usa la acción como estrategia de enseñanza-aprendizaje lleva implícita la reflexión continua sobre lo actuado, que permite mejorar la práctica futura; por lo tanto, no ocurre lo uno sin lo otro. En palabras de Paulo Freire: "No puede haber palabra verdadera que no sea un conjunto solidario de dos dimensiones indicotomizables, reflexión y acción". (Freire 1969, 17).

Frente al antropocentrismo de la educación ambiental tradicional, la experiencia descrita se acerca más a una visión biocéntrica, que significa colocar lo vivo como referencia de toda acción y conocimiento. Según Eduardo Gudynas, la visión biocéntrica rompe con la postura antropocéntrica de la modernidad, pues se deja de percibir a la naturaleza como un conjunto de objetos al servicio del ser humano y se le reconocen valores propios e incluso derechos (Gudynas 2011). La dicotomía antropocentrista ser humano-naturaleza también se rompe, pues dentro del biocentrismo el ser humano forma parte de la naturaleza; por lo tanto, no puede abstraerse de ella y pretender que es su benefactor. (Ferreira, Reis y de-Souza 2009).

La concepción de educación popular ambiental es más cercana a la experiencia que se reflexiona en este artículo. Según Juan Francisco Santos Estévez (2013), la educación ambiental es un proceso educativo que define nuevas formas de interacción con el mundo, actual y futuro, el cual permite alcanzar una calidad de vida superior, mediante la construcción de una sociedad que ame y respete todas las formas de vida.

Pensada como un proceso, la educación ambiental no es una línea recta de acumulación de conocimientos, más bien sigue el patrón de un aprendizaje que avanza en espiral. Para madurar, pasa muchas veces por el mismo punto $\mathrm{y}$, a veces, retrocede para reconstruir aprendizajes. Tampoco ocurre como un acto individual, sino colectivo. Como dice Paulo Freire: "Ahora, ya nadie educa a nadie, así como tampoco nadie se educa a sí mismo, las personas se educan en comunión". (Freire 1972, 61).

La educación en general, pero la ambiental en particular, necesita de espacios libres y liberadores, de contextos nuevos, que renueven la esperanza, la alegría, la emoción de aprender, y eso no ocurrirá dentro del encuadre de la educación tradicional. A continuación, se presenta y discute una experiencia de educación ambiental, que se construyó tejiendo, sin conceptos ni objetivos predefinidos. Esta sucedió en la Amazonía ecuatoriana y, más específicamente, en comunidades kichwas de la provincia de Napo, en la ciudad de 
Tena. Para que el lector pueda situar mejor la experiencia, se comienza con una descripción del contexto.

\section{Breve descripción del contexto de interés}

De los $12500 \mathrm{~km}^{2}$ que abarca la provincia de Napo, en Ecuador, 68\% se encuentra bajo categoría de conservación, legalmente reconocida. Más de 8000 $\mathrm{km}^{2}$ de territorio, ocupadas por diversos tipos de bosques: altimontanos por arriba de 3000 m s. n. m. y pantanosos por debajo de 400 m s. n. m.; una inmensidad verde conformada por diferentes ecosistemas que propician una rica diversidad de flora y fauna.

A la riqueza natural del paisaje se le suma la riqueza cultural de la provincia. De una población de 103697 habitantes, 56,75\% son indígenas, en su mayoría kichwas, pero hay presencia de unas 12 nacionalidades más. (GAD-Napo 2012).

La riqueza natural y cultural de la provincia contrasta con las presiones socioeconómicas que experimenta. El crecimiento urbano acelerado de Napo se nota en una demanda creciente de viviendas; a falta de un plan de crecimiento ordenado, se construyen viviendas en áreas de riesgo, en orillas de ríos y, continuamente, se ocupan áreas protegidas. A lo anterior se le suma el deficiente manejo de desechos y sus consecuencias en la contaminación de ríos, además de la pérdida de fuentes de agua.

Por su parte, crece la deforestación de bosques a causa de la extracción de madera y del cambio en el uso de los suelos; persiste una agricultura de subsistencia y una ganadería a pequeña escala, en una zona de suelos con vocación forestal y con pastizales de deficiente calidad para la crianza de ganado.

\section{Fotografía 1. Modelo tejido de serpiente coral (Micrurus surinamensis),} manduru machacuy en kichwa.

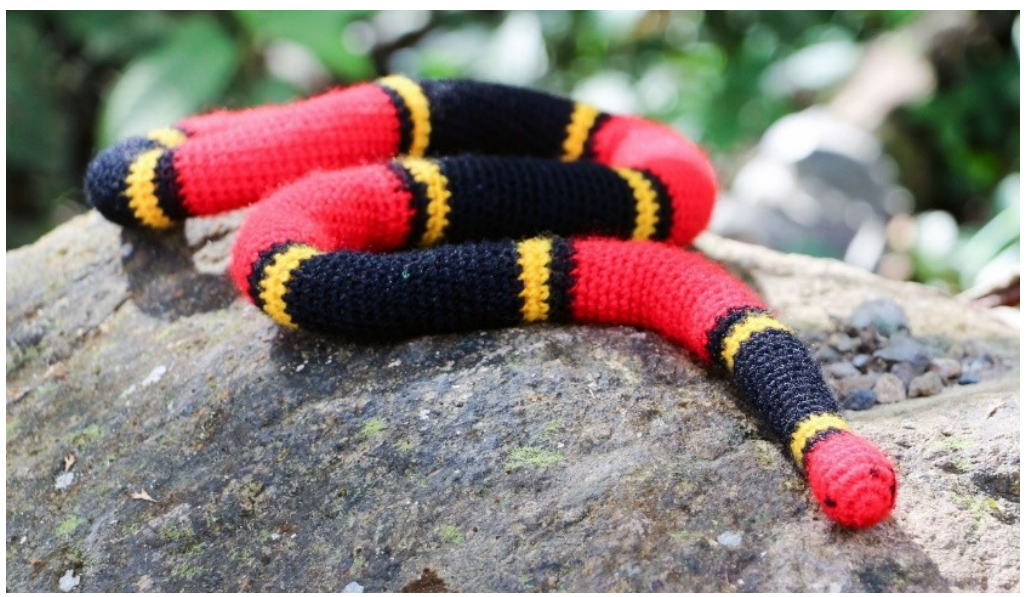

Fotografía propia. 
La zona de mayor interés para este artículo es la Reserva Biológica Colonso Chalupas, creada en 2014. Tiene una extensión de 93246 hectáreas, distribuidas entre los cantones de Archidona y Tena. La variedad climática y pluviométrica de la Reserva genera múltiples ecosistemas de bosque, con una rica diversidad de flora y fauna.

Según el Ministerio de Ambiente del Ecuador (MAE), en el área de interés, se podrían encontrar 171 familias de plantas, con 649 géneros y 1841 especies; de las cuales, 144 son especies endémicas. En cuanto a fauna, se ha detectado la presencia de diversidad de mamíferos: venados, murciélagos, armadillos, roedores, primates y felinos; se estima que podrían hallarse 10 órdenes, 30 familias y alrededor de 147 especies.

Respecto de la identificación de herpetofauna, por ahora, se registran 180 especies de anfibios. Con las aves ocurre otro tanto igual, se han identificado 119 especies, de 501 especies que, potencialmente, podrían encontrarse (MAE 2014). Según la misma fuente, solo $13 \%$ de la Reserva ha sido estudiada hasta el momento, por eso, las cifras podrían aumentar, de forma significativa, en los próximos años.

Los servicios ecosistémicos que proporciona la Reserva son diversos: aprovisiona de agua dulce a las ciudades de Tena y Archidona; abastece de alimentos, plantas medicinales y materias primas a los pobladores de comunidades cercanas; y, por si esto fuera poco, presta servicios de regulación hídrica y captura de dióxido de carbono. Finalmente, sirve de inspiración cultural para las comunidades y pone a su disposición atractivos turísticos y medios educativos. (MAE 2016).

Dentro de los límites territoriales de los dos cantones en que se encuentra la Reserva, habitan cerca de 58000 personas y, en la zona de amortiguamiento, existen 23 comunidades con una población aproximada de 4000 personas, de las cuales $83 \%$ vive en situación de pobreza. (Yaguache y otros 2016).

Las condiciones de pobreza en que viven esas comunidades son características de zonas rurales: poseen tierra, pero no los recursos para hacer uso productivo de ella; una ingesta alimenticia dependiente de productos cultivados por la familia; problemas socioeconómicos, como el alcoholismo y violencia intrafamiliar; pero, sobre todo, un abandono estatal manifiesto en la falta o precariedad de los servicios de educación, salud, empleo e infraestructura social. La suma de estas condiciones potencia y reproduce los problemas señalados, empobreciendo cada vez más a la población, tradicionalmente marginada y excluida.

En el año 2014, los autores de este artículo se pusieron en contacto con comunidades kichwas de Tena. Esto ocurrió en un momento de relevancia sociopolítica para la Amazonía, en que el Gobierno ecuatoriano estaba fundando una universidad pública en esta área.

La fundación de la Universidad Regional Amazónica Ikiam, que significa "selva" en idioma shuar, involucró la llegada de decenas de investigadores y de 
estudiantes, motivados por desarrollar proyectos con las comunidades cercanas. Uno de estos proyectos surgió de manera discreta y sin otra expectativa que tejer, junto a personas de las comunidades, como una actividad de acercamiento, de inmersión y de reconocimiento. Poco a poco, la idea fue madurando, tomando forma, hasta convertirse en un proyecto relevante para la universidad y con reconocimiento internacional. A continuación, se contará la historia resumida del proyecto y cómo se fue tejiendo punto a punto.

\section{Tejer para generar confianza}

El primer grupo de tejedoras estaba integrado por tres mujeres kichwas y la facilitadora. El vínculo que las unía era ser compañeras de trabajo en Ikiam. Se reunían fuera de la universidad a tejer y a conversar. Mientras tejían, los temas de conversación surgían espontáneamente; se hablaba de la familia, de los hijos, de la comunidad; eran reuniones de reconocimiento, mediadas por el tejido. No había ninguna dinámica de presentación, no se definían objetivos previos, no se seguía ninguna agenda; sin embargo, el tejido funcionaba como un articulador y creaba el ambiente propicio para conocerse.

La actividad del tejido exige un grado de concentración, habilidades psicomotrices finas que facilitan unir puntos, pensamiento lógico formal para contar y seguir patrones, entre otras cosas. No obstante, estas exigencias no fueron un obstáculo para la perseverancia y, poco a poco, surgieron las cadenas que unían puntos, que a su vez iban dando forma a objetos, combinando colores. En lugar de la frustración, surgía una sensación de ser capaz y con esta se potenciaba la confianza en la capacidad creadora.

Tal ambiente creativo generó un clima de relajamiento, en el cual la confianza emergió sin presiones. Paulatinamente, se dejaba de lado la timidez y con palabras se tejía una red que acercaba a las personas. Después del tercer encuentro, las mujeres ya estaban hablando de sí mismas, de lo que sentían, de lo que creían, algunas iban más lejos y hablaban de sus sueños, los de anoche, pero también esos que alumbran el camino que lleva al futuro deseado. El tejido acerca a las personas, genera y fortalece vínculos, articula relaciones, produce complicidades. Cuando se teje, se tejen espacios para hablar, para escucharse, para educarse de modo colectivo. Esto no es algo nuevo, hay muchas experiencias que lo han puesto en evidencia; la obra artística de María Angélica Medina es un buen ejemplo. Medina teje como un acto liberador, y cuando teje acompañada, emergen historias y relatos; según ella, porque tejer se vuelve un acto automático y, al ser automático, libera y da oportunidad de reflexionar sobre el diario vivir (Escobar 2007).

De acuerdo con Mariana Xochiquétzal, "el tejido tiene un poder para sanar, cohesionar, unir y organizar grupos humanos (...) este oficio en particular tiene características meditativas al ser repetitivo, introspectivo y (...) ayuda a despejar el pensamiento y aclarar ideas" (García Rivera 2017, 141). Según 
Rusiñol, Rodríguez y Ramón (2017), tejer tiene un potencial terapéutico, pues reduce la ansiedad y facilita la relajación, generando un ambiente propicio para la interiorización personal y el desarrollo de vínculos sociales saludables, entre muchas otras utilidades que destacan.

Viendo en retrospectiva la experiencia de la que nace este artículo, puede decirse que estas primeras reuniones y sus beneficios catapultaron lo que seguía: había que seguir tejiendo, pero en la universidad, para que más personas asistieran y ojalá el estudiantado se interesara; y este se involucró. Así surgió la primera versión organizada del proyecto.

\section{Tejer para educar ambientalmente}

En aquel tiempo, la universidad propiciaba el desarrollo de Proyectos Integradores de Saberes (PIS), acciones organizadas que un grupo de estudiantes debía ejecutar con la asesoría de un docente. La primera versión del proyecto proponía realizar un sondeo en las escuelas del sector, al respecto de la percepción que tenían los niños y las niñas sobre algunos animales amazónicos. Luego, se tejerían esos animales, se regresaría a las escuelas a desarrollar actividades lúdicas, y, a posteriori, se verificaría si las percepciones habían variado.

El stock de animales estaba integrado por animales que producían rechazo en la población local: la serpiente coral (Micrurus surinamensis; manduru machacuy en kichwa), y la "mal agüero" (Amphisbaenia fuliginosa; tapia machacuy en kichwa).

Los resultados del proyecto mostraron que, en sus fallas, estaba la semilla de su perfeccionamiento. Las maestras de las escuelas miraron con recelo que los niños socializaran, en el aula, con la réplica de un animal peligroso, pues luego se encontrarían con el animal verdadero en el bosque y podrían subestimar el riesgo de tomar contacto con él.

En otro orden de ideas, emergieron representaciones simbólicas que no se esperaban: la Amphisbaenia, que se asociaba con "mala suerte", los kichwas incluso rechazaban tocar el prototipo tejido. Lo primero ayudó a descubrir el error de sobreponer la percepción conservacionista, en un lugar donde prevalecía el conocimiento ancestral de riesgo de los animales venenosos, Lo segundo gatilló la utilidad ilimitada que podrían tener los animales tejidos para desarrollar educación ambiental.

En el caso concreto de la Amphisbaenia, la percepción kichwa asocia su aparición con "mala suerte", pues suele aparecer antes de temblores e inundaciones. El comportamiento de este animal más bien obedece a señales ambientales y geológicas, ya que alerta sobre desastres naturales. Esto muestra que, donde la ciencia tradicional observaría solo superstición, una ecología 
de saberes halla la oportunidad de combinar ciencia y conocimiento ancestral.

Los resultados del PIS se exhibieron en una casa abierta de la universidad y se dispuso que los animales tejidos se pondrían a la venta; los fondos recolectados servirían para darle continuidad al proyecto. Para ese entonces, el grupo de tejedores había crecido, pues las instalaciones de la universidad ofrecían más espacio, y así, se incorporaron estudiantes y personal de Ikiam.

En ese momento, ocurrieron tres cosas que marcarían la siguiente etapa del proyecto: a) si bien el grupo de tejedores era más numeroso, su asistencia no era constante, lo cual, sumado a las interferencias y distracciones del lugar, daba como resultado interacciones más superficiales; b) las lecciones aprendidas en el PIS sirvieron para descubrir el potencial de tejer animales amazónicos para ser usados como objetos de educación ambiental; y c) la venta de los prototipos fue exitosa y despertó una expectativa económica en algunas personas, lo cual marcaría la finalidad temporal de una de las etapas del proyecto.

Por un lado, de los incisos "b" y "c", se hablará en subtítulos específicos más adelante, ahora es importante reflexionar sobre las consecuencias que tuvo tejer-en-la-universidad. Por otro lado, las utilidades subjetivas de tejer, señaladas en el subtítulo anterior, el clima de confianza y la capacidad creadora emergen en las siguientes condiciones específicas.

La primera condición por respetar es la cantidad de participantes. La participación personalizada es óptima en grupos reducidos, de no más de 10 personas; podría ser hasta 20 personas en caso de tener un vínculo previo.

La segunda condición que se debe cuidar es la intimidad del lugar en donde se teje; por ejemplo, el ambiente del encuentro al tejer en la casa de una de las participantes era completamente distinto a tejer en la cafetería de la universidad.

La tercera condición es un desafío inherente al ambiente universitario: es posible que tejer-en-la-universidad evoque modelos tradicionales de enseñar y de aprender. La cooperación es sustituida por la competencia; la iniciativa, por la dependencia hacia el facilitador; y la creatividad, por la necesidad de un modelo de producción. Probablemente este sea uno de los problemas que origina la educación ambiental escolarizada, ya que genera un clima de enseñanza-aprendizaje reproductor de valores y de actitudes tradicionales, contrarios a los que se necesitan para crear una visión biocéntrica.

\section{Tejer como una actividad educativa}

Desde el momento que se decidió tejer animales amazónicos, una constante en el proyecto ha sido que no solo el objeto tejido sirve para educar, sino que tejer en sí mismo educa. La sensibilidad y la percepción de los animales 
cambia una vez que se reconoce su forma, su tamaño, sus colores, las características específicas que distinguen unos de otros. Estos contenidos, que se aprendieron de modo natural al tejer los animales, difícilmente podrían haber sido transmitidos mediante una charla educativa.

\section{Fotografía 2. Rana venenosa de líneas amarillas (Ranitomeya variabilis).}

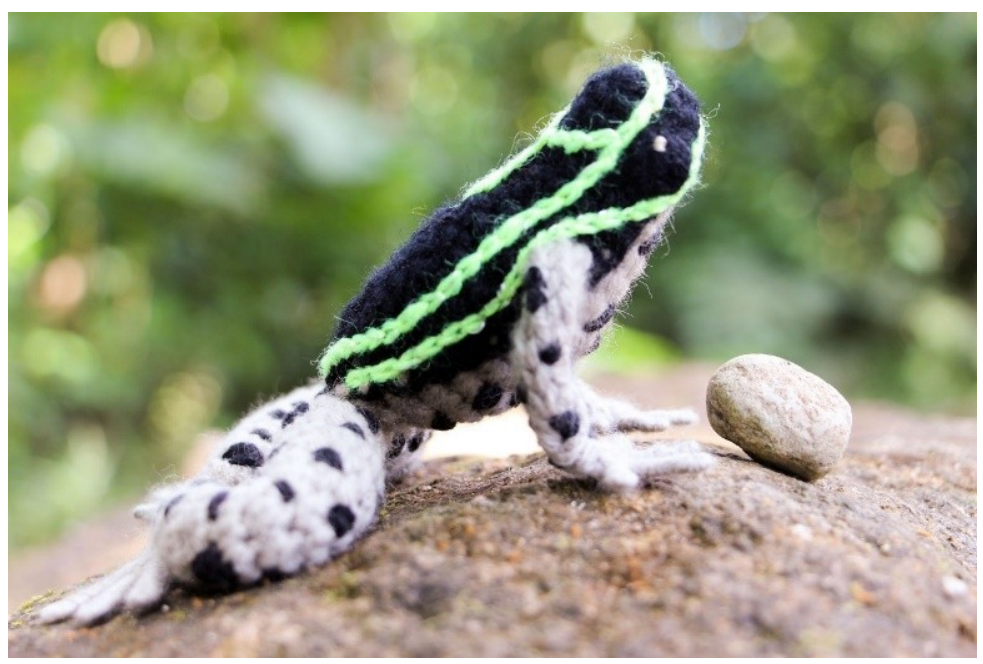

Fotografía propia.

Lo que se teje se vuelve propio, el valor de la capacidad creadora se deposita en el objeto terminado y, entonces, cuando vuelve a tener contacto con el objeto real, la percepción de este ha cambiado y surge una nueva sensibilidad: se le aprecia, se le reconoce, se le respeta.

Dicha experiencia ha sido descrita por las participantes que llevan más tiempo tejiendo: están redescubriendo la riqueza de lo que les rodea, una biodiversidad que desborda y, a veces, se mete por la puerta en forma de rana, mariposa y serpiente. Aun, los animales tejidos tienen un valor simbólico en sí mismos, pues son parte del imaginario kichwa, de su memoria e identidad cultural, de su relación con la naturaleza (Pérez 2017).

En la Amazonía, no hay forma de esconderse de las serpientes, por lo menos una vez a la semana se atravesarán en los caminos que conducen al cultivo familiar. Matarlas no es una solución viable, eso lo han entendido muy bien las tejedoras. La salida que ellas han asumido es conocerlas, distinguir a las verdaderamente peligrosas y cambiar la percepción que las une al ser humano; para ello, el tejido es una herramienta de educación ambiental sumamente útil. 


\section{El tejido como emprendimiento socioproductivo}

La primera experiencia de venta de animales tejidos fue exitosa: se vendieron todos y a precios atractivos. Esto despertó el interés de algunas tejedoras y del personal de la universidad que promocionaba el emprendimiento comunitario.

En ese momento, el proyecto fue conocido en más comunidades; por lo tanto, se multiplicaron las solicitudes de capacitación. Así, surgieron los talleres de tejido en las comunidades, los cuales ya llevaban aparejada la intención productiva. Muchas tejedoras habían asumido el reto de tejer animales amazónicos que se apegaran al modelo real, lo cual en ese momento se concebía como un valor agregado del producto.

En fin, el proyecto tomó un rumbo inesperado y lo que en un inicio fueron encuentros de tejedoras pasaron a ser sustituidos por talleres formativos. Incluso las instituciones públicas de economía popular y solidaria solicitaron capacitaciones para sus comunidades.

De la noche a la mañana, se descubrió el potencial productivo del proyecto, y como una forma de darle realce, se postuló como candidato a un premio internacional. Para bien o para mal, el proyecto fue reconocido dentro de los primeros lugares, recibió un premio monetario y beneficios consistentes en formación para sus participantes.

En este punto, las expectativas crecieron y amenazaron con desbordar la capacidad de la universidad para acompañar el proceso. También, las capacidades de las tejedoras se excedieron: se comenzó a tejer a toda velocidad para suplir la demanda, fue imposible mantener los estándares de exactitud biológica en los modelos y el proceso educativo no fue prioridad.

De manera apresurada, se presionó a las tejedoras a definir una marca para sus productos y a registrar el emprendimiento. Además, los llamados a asociarse legalmente para la producción comenzaron a sonar entre grupos. El hecho de constituir asociaciones productivas es una actividad promovida por el Estado ecuatoriano desde el 2011, cuando la Asamblea Nacional del Ecuador aprobó la Ley de Economía Popular y Solidaria y se crearon las instituciones que acompañarían el proceso. A partir de ese año, se han multiplicado las asociaciones comunitarias, pero en pocos casos se ha logrado la productividad autogestionada orientada por el Buen Vivir, sin fines de lucro o acumulación de capital, según artículo 1 de dicha ley (Asamblea Nacional del Ecuador 2011).

Las razones por las cuales el asociacionismo comunitario no se ha desarrollado como se esperaba son complejas, pero pueden resumirse en lo siguiente: a) las Asociaciones nacen con una estructura organizativa definida por la ley, que en muchos casos impone modelos autoritarios de liderazgo; b) las Asociaciones funcionan con excesiva burocracia, se multiplican las asambleas, los trámites y los oficios, pero no las actividades productivas; c) las or- 
ganizaciones públicas que acompañan a las asociaciones generan expectativas económicas, pero no desarrollan capacidades productivas y gerenciales para que funcionen; d) las condiciones de pobreza de los participantes hacen que vean en la asociación una salida económica a su situación, una fuente de empleo inmediata, y cuando esto no ocurre, se producen conflictos entre sus miembros, compiten por recursos escasos, se confrontan, etc.

Visto en retrospectiva, resulta interesante observar que, en esta etapa el proyecto, las tejedoras fueron urgidas por una demanda productiva; se descubrió la potencialidad de venta de los animales tejidos y prevaleció el interés de convertirlos en mercancía. En una escala mucho mayor, la educación ambiental tradicional reproduce este mismo interés mercantil en las relaciones ser humano-naturaleza. De acuerdo con Pablo Sessano (2014), esta relación es instrumental y concibe a la naturaleza como un conjunto de recursos disponibles para la productividad humana; según él, la educación se convierte en un proceso ineludiblemente funcional al modelo que ha generado los desastres ecológicos actuales, aun en su versión de educación ambiental para la sustentabilidad, en la cual no se ha superado este enfoque.

Para lograr una visión distinta al modelo productivo del desarrollo económico tradicional, hace falta desarrollar un enfoque biocéntrico. El camino estará lleno de tropiezos y de retrocesos; sin embargo, recorrerlo es la única salida posible.

Después de un año, el interés de las tejedoras de asociarse productivamente no se ha concretado. El desencanto de algunas y la persistencia de otras están produciendo una situación interesante: el proyecto está volviendo a sus orígenes, se está reconciliando con sus motivaciones originales y se está reconstruyendo.

\section{Fotografía 3. Ardilla roja norteña (Sciurus igniventris)}

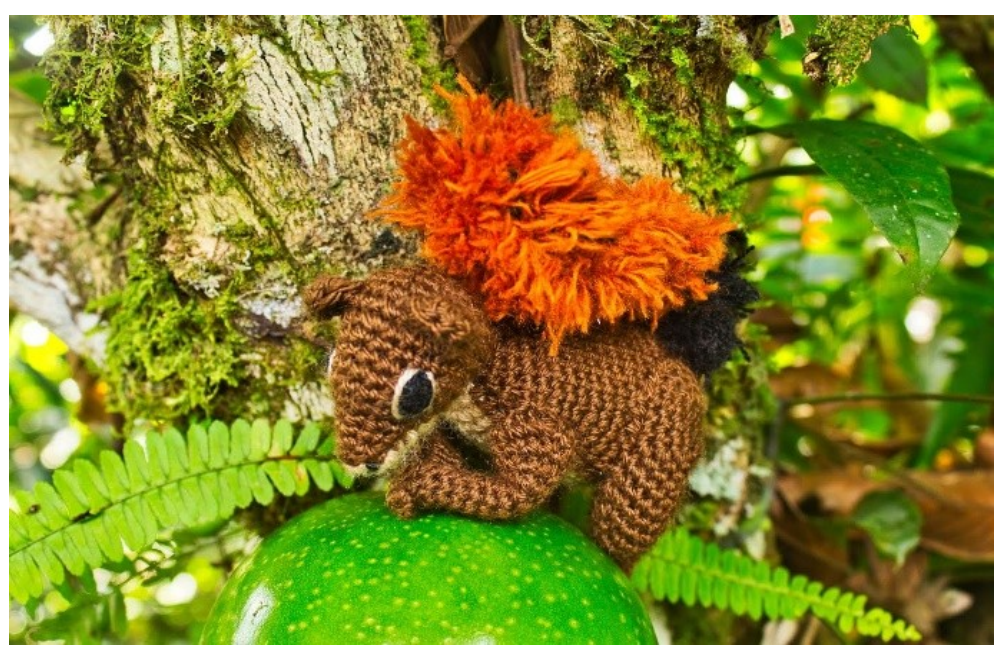

Fotografía propia. 


\section{¿Quiénes tejen?, ¿qué tejen?, ¿por qué tejen?}

Hasta ahora, las que han permanecido dentro del proyecto son mujeres; si bien algunos hombres se han acercado y muestran un interés inicial, pronto dejan de tejer. Las razones por las cuales tejen solo mujeres debe investigarse más en profundidad. Podría decirse que ellas tienen más tiempo o se organizan mejor para participar; también, podría ser que tejer se considera una actividad de mujeres, etc.

La presencia de hombres es más frecuente cuando el proyecto ha mostrado alguna posibilidad económica o cuando hay que responder a una entrevista de medios de comunicación y explicar las utilidades del tejido para la comunidad. En esos casos, hay hombres presentes; en los demás momentos, hay mujeres tejiendo.

\section{Fotografía 4. Grupo de tejedoras de la comunidad Alto Tena, con facilitadora Caroline Bacquet.}

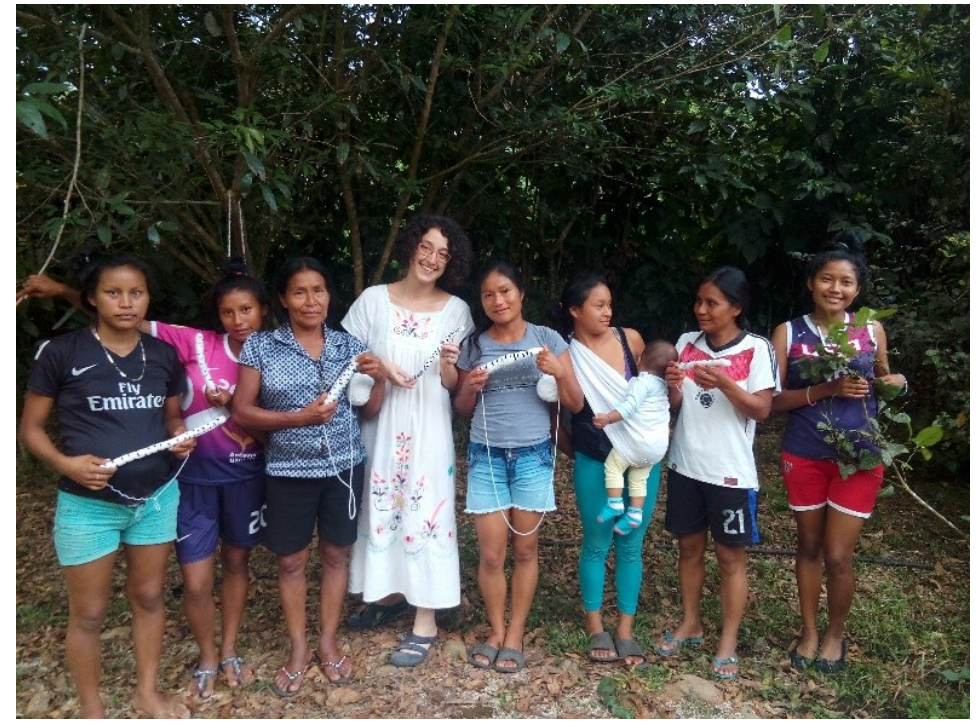

Fotografía propia

En términos objetivos, se tejen animales amazónicos, que, en la medida en que se apegan al modelo real, son instrumentos valiosos para la educación ambiental. Pero, en términos subjetivos, también se tejen nuevos vínculos, se tejen espacios de diálogo en los cuales se relatan historias que generan complicidades; se tejen percepciones y sensibilidades que renuevan el imaginario sociocultural del grupo. Se teje autoconfianza y sororidad, se tejen sueños de colores, con forma de animales sagrados. A medida que el proyecto avanza y madura, se teje la posibilidad de retomar la idea de asociarse productivamente. Ahora se sabe que, para llegar ahí, se debe tejer una larga cadena de 
puntos, de aprendizajes, de emociones, incluso de formación política, para que la prisa, el inmediatismo económico y los hombres no vuelvan a secuestrar lo que sale de las manos de las mujeres.

De los diálogos de las mujeres tejedoras, se puede inferir que tejen porque han encontrado que esta actividad les permite aportar creativamente a la educación ambiental. Tejen porque han descubierto que sus encuentros son un espacio que no despierta sospechas, un espacio de diálogos desapercibidos en el que, una a una, las palabras van tejiendo una nueva realidad.

Sin embargo, cuando se les pregunta, en el contexto de una entrevista formal, ¿por qué tejen?, muchas responden porque relaja, porque les gusta; la mayoría se ríen y dicen que tejen porque las distrae.

\section{Conclusiones}

Para concluir, se destacan cuatro temas discutidos en este artículo: a) tejer es una estrategia de enseñanza-aprendizaje idónea para educación y conservación ambiental; b) la educación y conservación ambiental están íntimamente ligadas al imaginario cultural de su contexto de referencia; c) el proyecto tiene posibilidades de constituir emprendimiento productivo, pero hay que trabajar las condiciones previas; d) los encuentros de tejido propician vínculos subjetivos entre mujeres que pueden aprovecharse para múltiples fines.

Como estrategia de educación y de conservación ambiental, el tejido tiene una doble utilidad: tejer animales es una experiencia que por sí misma cambia la percepción que se tenía de los animales tejidos; los animales tejidos son objetos didácticos que facilitan el conocimiento sensible de los animales del bosque.

Entonces, se contienen en una misma acción los aprendizajes prácticos de tejer, junto con nuevas percepciones y una sensibilidad renovada de lo tejido.

En segundo lugar, el proyecto ha mostrado la íntima relación entre la conservación de la biodiversidad y la diversidad cultural. Cuando se teje un animal amazónico, no se genera únicamente conciencia de conservación, también se recrea el imaginario simbólico que une la cultura con ese animal y, por lo tanto, aquello que une la cultura con la naturaleza.

De esa última cuentan las posibilidades de conservación de la Reserva Colonso Chalupas, las cuales están íntimamente ligadas a la riqueza cultural de las comunidades kichwas que se encuentran en su zona de amortiguamiento.

Una de las lecciones esenciales aprendidas hasta ahora en el proyecto ha sido que introducir, de forma prematura, la expectativa de comercializar los animales tejidos amenaza el proceso. Las participantes viven en condiciones 
económicas precarias, sus ingresos son exiguos y apenas son suficientes para resolver las necesidades cotidianas. Introducir una expectativa económica, sin las condiciones apropiadas, confronta el interés de aprender con las necesidades económicas inmediatas.

Antes de comercializar los productos, hay que lograr que las tejedoras mejoren sus habilidades técnicas, que aspiren a tejidos con valor artístico, productos que les hagan sentirse orgullosas y dignas, que honren el valor simbólico y cultural de lo que tejen.

Para finalizar, los encuentros de tejido se han constituido en espacios de diálogo entre mujeres; un lugar privilegiado para recopilar la voz de quienes no la tienen; y un espacio para contar y escribir historias que ayuden a entender mejor la situación de las mujeres kichwas. Desde esta modalidad de trabajo, podrían trabajarse múltiples temas: empoderamiento de las mujeres, sensibilización de género, incluso formación política.

Actualmente, se están generando sinergias con otros grupos de tejedoras, de realidades socioculturales distintas, lo cual abre la puerta a oportunidades ilimitadas de hacer trabajo sociocultural y, por qué no, de emprendimiento social.

El tejido es un trampolín, basta con pararse en la orilla de la inmensidad que convoca, tomarse de la mano con el grupo y lanzarse, confiando en que la red que se ha tejido ayudará a volar y, si toca caer, esa misma red amortiguará la caída.

\section{Bibliografía}

Breiting, Soren. 1997. Hacia un nuevo concepto de Educación Ambiental. Carpeta informativa del CENEAM. Madrid, España: Ministerio de Ambiente, 1997.

Caride, José Antonio y Pablo Ángel. Meira. 2003. Educación Ambiental y desarrollo humano. Barcelona, España: Ariel, 2003.

Ley de Economía Popular y Solidaria, de Asamblea Nacional Constituyente. 2011. Ley de Economía Popular y Solidaria;. Quito, Ecuador 2011.: Asamble Nacional Constituyente, República del Ecuador.

Escobar, Ángela Patricia. 2007. «Tejer como protesta». Conferencia pronunicada en Medellin, Colombia: Encuentro Internacional Medellin 2007. Prácticas Artísticas Contemporáneas, 11 de febrero.

Ferreira, Nanci, Sonia Reis y María de-Souza. 2009. «Educación biocéntrica: pedagogía del afecto». Pensamiento Biocéntrico, julio-diciembre: 67 a 80 . 
Fogar, Mariela. 2013. «La modernidad como forma de conocer y ser en el mundo» Corrientes del pensamiento contemporáneo. Materiales de cátedra. Argentina: Universidad Nacional del Nordeste, Facultad de Humanidades, Departamento de Ciencias de la Educación.

Freire, Paulo. 1969. La educación como práctica de libertad. Monte Video Uruguay: Tierra Nueva.

Freire, Paulo. 2004. Pedagogía de la autonomía. Saou Paulo, Brasil: Paz e Terra SA.

Freire, Paulo. 1972. Pedagogía del oprimido. Montevideo, Uruguay: Siglo XXI Editores.

GAD-Napo. 2012. «PDyOT Napo 2020». Tena, Napo - Región Amazónica del Ecuador.

Garcia Rivera, Mariana Xochiquétzal. 2017. «Tejer y resistir. Etnografías audiovisuales y narrativas textiles» UNIVERSITAS Revista de Ciencias Sociales y Humanas: $n^{\circ}$ (27), julio a diciembre 2017: 141- a 160.

García, Eduardo. 2002. «Los problemas de la educación ambiental: ¿Es posible una educación ambiental integradora?» Editado por Centro Nacional de Educación Ambiental. Investigación en la Escuela, ( $\left.n^{\circ} 46\right)$.

González Gaudiano, Edgar y Miguel Ángel Arias Ortega. 2009. «La educación ambiental institucionalizada: actos fallidos y horizontes de posibilidad» Editado por IISUE-UNAM. Perfiles Educativos XXXI, (Madrid, España, no 124), noviembre 2009.

Gudynas, Eduardo. 2011. «Debates sobre el desarrollo y sus alternativas en América Latina: Una breve guía heterodoxa.» En Mas allá del desarrollo, de Miriam Lang y Dunia Mokrani:, 21- a 54. Quito, Ecuador: Abya Yala.

Gutiérrez Pérez, José. 1995. La Educación Ambiental. Fundamentos teóricos, propuestas de transversalidad y orientaciones extracurriculares. Madrid, España: La Muralla, 1995.

MAE (Ministerio de Ambiente). 2014. Estudio de alternativas de manejo para la declaratoria de área protegida al bosque protector "cuencua de los ríos Colonso, Tena, Shitih e Inchillanqui" y el área del patrimonio forestal del estado unidad 2. Tena, Ecuador: MAEinisterio de Ambiente.

MAE (Ministerio de Ambiente). 2016. Plan de Manejo de la Reserva Biológica Colonso Chalupas. Tena-Ecuador: MAE, IKIAM, GIZ.

ONU (Organización de las Naciones Unidades). 1972. «Conferencia de Naciones Unidas sobre el Medio Humano». Estocolmo, Suecia: Organización de las Naciones Unidas ONU.

Pérez, Annabella Ponce. 2017. «Tejido como relato social». Tesis de mMaestría en Diseño., Facultad de Diseño y Comunicación de la Buenos Aires, Angentina: Universidad de Palermo, Facultad de Diseño y ComunicaciónBuenos Aires, Argentina. 
Rusiñol Rodríguez, Judit, María Rodríguez Bailón y Anna Ramón Aribau. 2017. «Motivos, efectos y beneficios terapéuticos de la actividad de hacer punto: Una revisión de la literatura» TOG A Coruña: 217 a 231.

Santos Estévez, Juan Francisco. 2013. «¿Qué entendemos por educación ambiental?». En Un tejido de muchos puntos. Compilación sobre educación popular ambiental, de Bettty Hernández Bercera y Rosa Pablos de la Rosa:, 11- a 17. La Habana-Cuba: Camino.

Sessano, Pablo. 2014. «Praxis educativa, espacio político y alfabetización ambiental: el desafío de los educadores ambientales frente al imperativo, en clave colonialidad/decolonialidad». En La educación ambiental en la Argentina: actores, conflictos y políticas públicas, de Telais Aldana, María Laura Canciani, Pablo Sessano, Sandra Alvino y Ana Padawer, 89- a 111. Buenos Aires, Argentina: La Bicicleta Ediciones.

UNESCO (United Nations Educational Scientific and Cultural Organization). 1987. «Congreso Internacional de Educación Ambiental y Formación sobre el Medio Ambiente». Moscu, RUSIA: United Nations Educational Scientific and Cultural Organization.UNESCO.

UNESCO (United Nations Educational Scientific and Cultural Organization). 2017. «Educación para los objetivos de desarrollo sostenible». Paris, Francia: United Nations Educational Scientific and Cultural OrganizationUNESCO.

Yaguache, Roberto, Lizbeth Andi, Valerio Andi y Jaime Tanguila. 2016. Diagnóstico socioeconómico y ambiental en las comunidades del áea de amortiguamiento de la Reserva Biológica Colonso Chalupas. Quito, Ecuador: UNESCO-Ikiam, Cooperación Española. 